MATHEMATICS OF COMPUTATION

Volume 78, Number 268, October 2009, Pages 2427-2433

S $0025-5718(09) 02238-8$

Article electronically published on May 1, 2009

\title{
ALGEBRAIC SOLUTIONS OF JACOBI EQUATIONS
}

\author{
S. C. COUTINHO AND MARCOS DA SILVA FERREIRA
}

\begin{abstract}
We propose an algorithm to compute exactly the algebraic solutions of Jacobi equations over the projective plane.
\end{abstract}

\section{INTRODUCTION}

From the perspective of present day mathematics, a Jacobi equation is the Pfaff equation that corresponds to a vector field over the projective plane; see section 2 for a formal definition. These equations were named after Jacobi because he was the first to study them systematically in a paper [9] in 1842. In an article [5] in 1878, G. Darboux used Jacobi equations to illustrate his method of solving first order differential equations. As part of that he introduced the problem of computing the algebraic solutions of a Jacobi equation. In this guise, the equations became a standard topic in textbooks of the late 19th and early 20th centuries, like Jordan's Cours d'Analyse de l'École Polytechnique [10, p. 38], and Ince's Ordinary Differential Equations [8, p. 22].

In the late 1970s, Jouanolou reworked and improved Darboux's results using the language of linear algebra and algebraic geometry, [11 pp. 1-19]. In Jouanolou's approach one associates a matrix $B$ to a given Jacobi equation $\Omega$. The linear solutions of $\Omega$ can then be computed from the eigenvectors of $B^{t}$. Moreover, the nonlinear solutions, when they exist, can also be computed from $B$.

In this paper we investigate Jouanolou's approach to Jacobi equations from the point of view of computer algebra. Our interest in this problem is twofold. On the one hand, we want to use the Galois group techniques developed in 4] in order to give an efficient algorithm that can be used to compute first integrals of polynomial vector fields over the projective plane. On the other hand, as shown in $\underline{3}$, the existence of an algebraic solution implies that any nonholonomic $\mathcal{D}$-module defined as a deformation of a vector field by a polynomial function always has a holonomic quotient module. This, in turn, allows one to apply the algorithms of [12] to give estimates for the dimension of some Ext-groups involving these modules.

Since the algorithms based in [11, pp. 1-19] require the computation of eigenvalues and eigenvectors, it looks as if we have to work over an algebraic extension of the base field $\mathbb{K}$. However, our main result is just that this is not really necessary. Indeed, one can arrange all the algorithms so that both the output and all the

Received by the editor April 3, 2006 and, in revised form, April 23, 2008.

2000 Mathematics Subject Classification. Primary 34M15, 68W30; Secondary 13P10.

Key words and phrases. Jacobi equation, Gröbner bases, algebraic solutions.

During the preparation of this paper the first author was partially supported by grants from CNPq and PRONEX(ALGA).

The second author was partially supported by a scholarship from CNPq. 
computations are expressed directly in terms of $\mathbb{K}$ itself, without relying on field extensions.

This note is divided into four sections: after recalling some basic definitions from [11, pp. 1-8], we discuss algorithms to compute the linear solutions (section 3) and the nonlinear solutions (section 4) of a Jacobi equation. All the algorithms introduced here are implemented in the computer algebra system SINGULAR, see [7] and 6, and can be downloaded from http://www.dcc.ufrj.br/ collier/folia.html.

\section{Preliminaries}

A Jacobi form is a 1 -form $\Omega=P d x+Q d y+R d z$, where $P, Q$, and $R$ are homogeneous polynomials of degree two in $\mathbb{C}[x, y, z]$ that satisfy $x P+y Q+z R=0$. A Jacobi form is saturated if $\operatorname{gcd}(P, Q, R)=1$. A homogeneous polynomial $F$ of degree $k>0$ is an algebraic solution of $\Omega$ if $\Omega \wedge d F=\eta F$, for some homogeneous linear 2-form $\eta$.

It follows from the exactness of the Koszul complex [11, p. 2] that there exist linear homogeneous polynomials $L, M$, and $N$ such that

$$
P=z M-y N, \quad Q=x N-z L, \text { and } R=y L-x M .
$$

Of course, the triple $(L, M, N)$ is not unique. Indeed, two such triples differ by a constant multiple of $(x, y, z)$. Following Jouanolou we say that a triple is canonical if

$$
\frac{\partial L}{\partial x}+\frac{\partial M}{\partial y}+\frac{\partial N}{\partial z}=0 .
$$

Since $L, M$, and $N$ are linear, we may write $(L, M, N)^{t}=B(x, y, z)^{t}$ for some complex $3 \times 3$ matrix $B$. Moreover, it is easy to show that the triple $(L, M, N)$ is canonical if and only if $B$ has zero trace, [11, p. 9]. Thus, we have an isomorphism between the vector space of all Jacobi forms and the Lie algebra $\mathfrak{s l}_{3}(\mathbb{C})$, of $3 \times 3$ matrices of trace zero. It follows from [11, Lemme 2.2, p. 9] that if $u: \mathbb{C}^{3} \rightarrow \mathbb{C}^{3}$ is the linear transformation induced by a matrix $U \in \mathrm{SL}_{3}(\mathbb{C})$, then $U^{-1} B U$ is the matrix associated to the 1 -form $u^{*}(\Omega)$, where $B$ is the canonical matrix of $\Omega$. In particular, whenever convenient, we may take the canonical matrix $B$ of a given Jacobi form $\Omega$ to be in its Jordan canonical form. We assume, throughout this note, that $\Omega$ has coefficients in an effective field $\mathbb{K}$; which means that the arithmetic operations of addition, subtraction, multiplication, division, and the basic comparison required to determine equality between two elements of $\mathbb{K}$ can be done by algorithms; see [2]. In particular, $B \in \mathfrak{s l}_{3}(\mathbb{K})$.

\section{LinEAR SOLUTIONS}

We begin by recalling the method used in [11, $\$ 2.4$, p. 11] to determine the linear solutions of a Jacobi form. If $F=a x+b y+c z$ is such a solution, then

$$
\Omega \wedge d F=a L+b M+c N \text {. }
$$

Thus, $F$ is an algebraic linear solution of $\Omega$ if and only if $a L+b M+c N=\lambda F$, for some $\lambda \in \mathbb{C}$. This equation may be rewritten in matrix form as $B^{t} v^{t}=\lambda v^{t}$, where $v=(a, b, c)$. Therefore, the linear solutions of $\Omega$ correspond to the eigenvectors of $B^{t}$.

Since we are assuming that $B$ has coefficients in $\mathbb{K}$, it follows that the characteristic polynomial $\chi=\chi_{\Omega}$ of $B$ belongs to $\mathbb{K}[t]$. There are two cases, depending on whether $\chi$ has repeated roots or not. First, if $\chi$ has repeated roots, then they 
must all belong to $\mathbb{K}$. Indeed, if $B$ has fewer than three distinct eigenvalues, then $\operatorname{gcd}\left(\chi, \chi^{\prime}\right)$ is a nonconstant polynomial with coefficients in $\mathbb{K}$, where $\chi^{\prime}$ is the derivative of $\chi$ with respect to $t$. Moreover, $\operatorname{gcd}\left(\chi, \chi^{\prime}\right)$ will have degree one or two. In the former case it is clear that this root must be in $\mathbb{K}$. However, this is also true in the latter case, since the repeated root must appear with multiplicity two in the greatest common divisor. Therefore, if $\chi$ has repeated roots, then they all belong to the base field, and the eigenvectors can be found by solving a linear system over $\mathbb{K}$.

This leaves us with the case in which $\chi$ has three distinct roots. If these roots are in $\mathbb{K}$, they may be handled as above; otherwise, we proceed as follows. Let $q$ be the nonlinear irreducible factor of $p$. Since $\operatorname{deg}(\chi)=3$, it follows that $q$ has degree 2 or 3 .

Let $I$ be the ideal of $\mathbb{K}\left[t, u_{1}, u_{2}, u_{3}\right]$ generated by $q \in \mathbb{K}[t]$, and the three linear equations corresponding to the system $\left(B^{t}-t I\right) u=0$, where $u=\left(u_{1}, u_{2}, u_{3}\right)$. Now compute the reduced Gröbner basis $G$ of $I$ with respect to the lexicographical order which has $u_{1}>u_{2}>u_{3}>t$; see [1, sections 1.7 and 1.8]. It is easy to see that $G$ contains $q$ together with two elements of the form $g_{i} u_{i}-g_{j} u_{j}$ and $h_{i} u_{i}-h_{k} u_{k}$, for $1 \leq i, j, k \leq 3$, where the $g$ 's and $h$ 's are polynomials in $\mathbb{K}[t]$.

Recall that we are assuming that all the eigenvalues of $B$ are distinct, which implies that each eigenspace has dimension exactly one. In particular, $g_{i}=g_{j}=0$, $h_{i}=h_{j}=0$, and $g_{j}=h_{k}=0$ cannot occur because, in all three cases, the resulting eigenspaces would have dimension two. We analyse the remaining cases. First, if $g_{j} h_{k} \neq 0$, then $u_{j}=g_{i} u_{i} / g_{j}$ and $u_{k}=h_{i} u_{i} / h_{k}$. Taking $u_{i}=1$ we find that the eigenvector is given by $u_{j}=g_{i} / g_{j}$ and $u_{k}=h_{i} / h_{k}$. Note that it may happen that $u_{k}=0$ or $u_{j}=0$. On the other hand, if $g_{j} \neq 0$ and $h_{k}=0$, we have that $h_{i} \neq 0$, so that $u_{i}=0$ and $u_{j}=g_{i} u_{i} / g_{j}=0$. The case $h_{k} \neq 0$ and $g_{j}=0$ is entirely analogous. Finally, if $v$ is an eigenvector of $B^{t}$, then the desired linear solution is given by the inner product $v \cdot(x, y, z)^{t}$.

This reasoning can be immediately turned into an algorithm that returns the linear solutions as a function of $t$, where $t$ is one of the roots of the characteristic polynomial $\chi$. As an inevitable consequence of this we have that the linear solutions may not have coefficients in $\mathbb{K}$. However, we can still package them as a polynomial in $\mathbb{K}$. This is a consequence of the following proposition.

Proposition 3.1. Let $\Omega$ be a Jacobi equation with coefficients in $\mathbb{K}$, and let $\chi=\chi_{\Omega}$ be irreducible over $\mathbb{K}$. If $\ell_{1}, \ell_{2}$, and $\ell_{3}$ are the linear solutions of $\Omega$, then $\ell_{1} \ell_{2} \ell_{3}$ is an irreducible polynomial in $\mathbb{K}[x, y, z]$.

Proof. First of all, it follows from the algorithm discussed above that each $\ell_{j}$ can be written in the form $\ell_{j}(\lambda, x, y, z)=a_{j} x+b_{j} y+c_{j} z$, where $a_{j}, b_{j}, c_{j} \in \mathbb{K}[\lambda]$, for some root $\lambda$ of $\chi$. Now if $\sigma$ is an automorphism in the Galois group $G$ of $\chi$ over $\mathbb{K}$, then $\sigma\left(\ell_{j}(\lambda, x, y, z)\right)=\ell_{j}(\sigma(\lambda), x, y, z)$, which is also a linear solution of $\Omega$ since this form is invariant under the pullback by $\sigma$. Set $F=\ell_{1}(\lambda) \ell_{2}(\lambda) \ell_{3}(\lambda)$.

Since $\chi$ is irreducible over $\mathbb{K}$, the Galois group $G$ is isomorphic either to the cyclic group $C_{3}$, or to the symmetric group $S_{3}$. In both cases $G$ contains an element $\rho$ of order 3 . Since $\rho$ permutes the three roots of $\chi$, it follows that

$$
F=\ell_{1}(\lambda)\left(\ell_{1}(\lambda)\right)^{\rho}\left(\ell_{1}(\lambda)\right)^{\rho^{2}}=\ell_{1}(\lambda)\left(\ell_{1}(\rho(\lambda))\right)\left(\ell_{1}\left(\rho^{2}(\lambda)\right)\right)
$$

is invariant under the subgroup generated by $\rho$. If the Galois group of $\chi$ is isomorphic to $C_{3}$, then this is enough to show that $F$ has coefficients in $\mathbb{K}$. 
Suppose now that $G \cong S_{3}$. Let $\tau$ be a transposition in this group. Then, $F^{\tau}=\ell_{1}^{\tau}(\lambda)\left(\ell_{1}(\lambda)\right)^{\tau \rho}\left(\ell_{1}(\lambda)\right)^{\tau \rho^{2}}$. However, $\tau \rho=\rho^{2} \tau$, so that

$$
F^{\tau}=\ell_{1}^{\tau}(\lambda)\left(\ell_{1}(\lambda)\right)^{\rho^{2} \tau}\left(\ell_{1}(\lambda)\right)^{\rho \tau}=\ell_{1}(\tau(\lambda))\left(\ell_{1}(\tau(\lambda))\right)^{\rho^{2}}\left(\ell_{1}(\tau(\lambda))\right)^{\rho} .
$$

Since $\tau(\lambda)$ is also a root of $\chi$, it follows that $F^{\tau}=F$. But $\rho$ and $\tau$ generate $G$, so $F$ must be invariant under all the elements of the Galois group. Therefore, $F \in \mathbb{K}[x, y, z]$.

A similar result holds for the product of two linear solutions if $\chi$ has an irreducible factor of degree 2 over $\mathbb{K}$. This suggests an algorithm which returns

(1) $\ell_{1}, \ell_{2}$, and $\ell_{3}$ if the three roots of $\chi$ belong to $\mathbb{K}$;

(2) $\ell_{1} \ell_{2}(\bmod q)$ and $\ell_{3}$ if $\chi$ has only one root in $\mathbb{K}$, and $q$ is its irreducible factor of degree 2 ;

(3) $\ell_{1} \ell_{2} \ell_{3}(\bmod \chi)$ if $\chi$ is irreducible over $\mathbb{K}$.

Note that in (2), the linear solutions $\ell_{1}$ and $\ell_{2}$ correspond to the roots of the irreducible factor $q$ of $\chi$.

One way to compute these polynomials is to find the linear factors, multiply them, and reduce the result modulo the irreducible factor of $\chi$ of degree 2 or 3 , as the case may be. However, the Gröbner machine of Singular can actually compute the resulting polynomial for a $3 \times 3$ matrix $B$ of trace zero with undetermined coefficients. If we consider the solution as a polynomial in the (eight) coefficients of $B$, together with $x, y$, and $z$, we find that it has degree 6 and 68 terms. For this reason, this is not a very practicable formula. A program that computes this polynomial can be found at the web page mentioned in section 1 .

\section{NONLinear SOLUTiOns}

Let $B$ be the matrix canonically associated to a Jacobi form $\Omega$. Denote by $J$ the Jordan canonical form of $B$ and by $\phi$ the linear change of coordinates that turns $J$ into $B$. As in the previous section, we have to analyse several cases, which will depend on the shape of $J$.

Suppose first that $J$ has three distinct eigenvalues. Applying $\phi^{*}$ to $\Omega$ we get

$$
\widehat{\Omega}=\phi^{*}(\Omega)=\left(\lambda_{1}-\lambda_{2}\right) y z d x+\left(\lambda_{2}-\lambda_{0}\right) x z d y+\left(\lambda_{0}-\lambda_{1}\right) x y d z,
$$

where $J=\operatorname{diag}\left(\lambda_{0}, \lambda_{1}, \lambda_{2}\right)$. Since $\widehat{\Omega}$ is saturated, its linear solutions, in the above coordinates, are $x, y$ and $z$. Thus, it follows from [11, Proposition 3.7.8, p. 120] that $\Omega$ has a finite number of algebraic solutions if and only if one of the ratios,

$$
\rho_{1}=\frac{\lambda_{0}-\lambda_{1}}{\lambda_{2}-\lambda_{0}} \text { or } \rho_{2}=\frac{\lambda_{0}-\lambda_{1}}{\lambda_{1}-\lambda_{2}}
$$

is an irrational number. Moreover, if one of these ratios is indeed irrational, then by [11, Théorème 3.3, p. 102] $\Omega$ has at most three solutions, namely the linear ones, already computed in section 3 . The next theorem sums up all that we need to know to turn the previous statement into an algorithm.

Theorem 4.1. If $\rho_{1}$ and $\rho_{2}$ are rational numbers, then either

(1) $\chi=\chi_{\Omega}$ is completely reducible over $\mathbb{K}$, or

(2) $\chi=t\left(t^{2}-\alpha\right)$, where $\alpha$ is not a perfect square in $\mathbb{K}$.

Moreover, in the second case, $\rho_{1}=\rho_{2}=-2$. 
Proof. Since we are assuming that both $\rho_{1}$ and $\rho_{2}$ are rational numbers, we may write

$$
\lambda_{0}-\lambda_{1}=r \theta, \quad \lambda_{1}-\lambda_{2}=p \theta, \text { and } \quad \lambda_{2}-\lambda_{0}=q \theta,
$$

where $p, q, r \in \mathbb{Z}$ and $\theta \in \overline{\mathbb{K}}$, the algebraic closure of $\mathbb{K}$.

Suppose, first, that none of the roots of $\chi$ is in $\mathbb{K}$. Since $\chi$ has degree 3 , it follows that it is irreducible over $\mathbb{K}$. Thus, its Galois group $G$ over $\mathbb{K}$ is isomorphic to $S_{3}$ or to $C_{3}$. In either case, there exists an automorphism $\sigma \in G$ such that $\sigma\left(\lambda_{i}\right)=\lambda_{i+1}$, where the sum of subscripts is computed modulo 3. In particular,

$$
r \sigma(\theta)=\sigma\left(\lambda_{0}-\lambda_{1}\right)=\lambda_{1}-\lambda_{2}=p \theta .
$$

Therefore, $\sigma(\theta)=p \theta / r$, which implies that $\sigma^{3}(\theta)=p^{3} \theta / r^{3}$. However, $\sigma$ has order 3 in $G$, so that $\sigma^{3}(\theta)=\theta$. Moreover, we may assume that $\theta \neq 0$ for, otherwise, $\chi$ would have repeated roots. Thus, $p^{3}=r^{3}$. Since $p$ and $r$ are integers, we conclude that $p=r$. But, this implies that $\lambda_{0}-\lambda_{1}=\lambda_{1}-\lambda_{2}$. Moreover, since $\lambda_{0}, \lambda_{1}$ and $\lambda_{2}$ are eigenvalues of a trace zero matrix, it follows that the $\lambda_{0}+\lambda_{1}+\lambda_{2}=0$. Putting these two facts together, we conclude that $\lambda_{1}=0$, which contradicts the irreducibility of $\chi$ over $\mathbb{K}$. Thus, we may assume, without loss of generality, that $\lambda_{2} \in \mathbb{K}$. It then follows from (4.1) that

$$
\lambda_{0}-\lambda_{2}=-\frac{\lambda_{0}-\lambda_{1}}{\rho_{1}} \text { and } \lambda_{1}-\lambda_{2}=\frac{\lambda_{0}-\lambda_{1}}{\rho_{2}} .
$$

Hence,

$$
\left(\lambda_{0}-\lambda_{1}\right)\left(\frac{1}{\rho_{2}}-\frac{1}{\rho_{1}}\right)=\lambda_{0}+\lambda_{1}-2 \lambda_{2} .
$$

However, $\lambda_{0}$ and $\lambda_{1}$ are the two roots of a quadratic equation with coefficients in $\mathbb{K}$. Thus, $\lambda_{0}+\lambda_{1} \in \mathbb{K}$. But, $\lambda_{2} \in \mathbb{K}$ by the hypotheses. Therefore, if $\rho_{2} \neq \rho_{1}$, then it follows from (4.3) that $\lambda_{0}-\lambda_{1} \in \mathbb{K}$. This implies that $\lambda_{0}$ and $\lambda_{1}$ belong to $\mathbb{K}$, so $\chi$ is completely reducible over $\mathbb{K}$, in this case.

Assume now that $\rho_{1}=\rho_{2}=\rho \in \mathbb{K}$. By (4.3), $\lambda_{0}+\lambda_{1}=2 \lambda_{2}$, so that

$$
0=\lambda_{0}+\lambda_{1}+\lambda_{2}=3 \lambda_{2} \text {. }
$$

Thus, $\lambda_{2}=0$, which implies that $\lambda_{1}=-\lambda_{0}$. Hence, $\rho_{2}=\rho_{1}=-2$, and the proof is complete.

Let us recall how the nonlinear solutions are obtained when $\rho_{1}$ and $\rho_{2}$ are both rational. Set $\rho_{1}=q_{2} / q_{1}$ and $\rho_{2}=q_{2} / q_{0}$, where $q_{0}, q_{1}$ and $q_{2}$ are pairwise co-prime integers. As in section $3, \ell_{i}$ denotes the linear solution that corresponds to the eigenvalue $\lambda_{i}$.

From the definition of the $\rho$ 's we have that two among them must have the same sign, whilst the third has the opposite sign. Moreover, we may assume, without loss of generality, that two among them are positive. Thus, if $q_{i}, q_{j} \geq 1$, then $q_{k}<0$ and by [11, p. 13] the nonlinear solutions of $\Omega$ have the form

$$
c_{1} \ell_{i}^{q_{i}} \ell_{j}^{q_{j}}+c_{2} \ell_{k}^{-q_{k}} \text {, where } c_{1}, c_{2} \in \mathbb{K} \text { and }\{i, j, k\}=\{1,2,3\} .
$$

Assume now that $\chi$ is not completely reducible over $\mathbb{K}$. By Theorem 4.1, one of the eigenvalues of $B$, say $\lambda_{k}$, must be zero. In this case, we may choose $q_{i}=q_{j}=1$ and $q_{k}=-2$. Therefore, the nonlinear solutions take the form

$$
c_{1} \ell_{i} \ell_{j}+c_{2} \ell_{k}^{2}, \text { where } c_{1}, c_{2} \in \mathbb{K} .
$$


However, we are assuming that $\chi$ has an irreducible factor of degree two, whose roots are $\lambda_{i}$ and $\lambda_{j}$. Thus, as pointed out after Proposition 3.1, $\ell_{i} \ell_{j}$ and $\ell_{k}$ have coefficients in $\mathbb{K}$. This leads to the following algorithm.

Algorithm. Given a Jacobi form $\Omega$ with coefficients in $\mathbb{K}$, whose canonical matrix has three distinct eigenvalues, the algorithm returns the nonlinear solutions of $\Omega$, if it has any.

Step 1: If $\chi$ is irreducible over $\mathbb{K}$, return all solutions are linear and stop.

Step 2: If $\chi$ is completely reducible over $\mathbb{K}$, compute its roots $\lambda_{0}, \lambda_{1}$ and $\lambda_{2}$; otherwise go to Step 5.

Step 3: Compute the reduced fractions $\rho_{1}=q_{3} / q_{1}$ and $\rho_{2}=q_{3} / q_{2}$, defined in (4.1).

Step 4: Find the linear solutions $\ell_{j}, 1 \leq j \leq 3$, of $\Omega$ using the algorithm of the previous section, and go to Step $\%$.

Step 5: If $\chi$ is not completely reducible over $\mathbb{K}$ and $\lambda_{k}=0$, let $q_{i}=q_{j}=1$ and $q_{k}=-2$.

Step 6: Compute $\ell_{i} \ell_{j}$ and $\ell_{k}$ using the algorithm of the previous section.

Step 7: If $q_{i}, q_{j} \geq 1$ and $q_{k}<0$, return $c_{1} \ell_{i}^{q_{i}} \ell_{j}^{q_{j}}+c_{2} \ell_{k}^{-q_{k}}$, where $c_{1}$ and $c_{2}$ are parameters, and stop.

From the computational point of view, this is the only interesting case: all the other cases are straightforward. We sum them up below. For more details, see [11, pp. 14-17].

First of all, if $B=\lambda I$, where $I$ is the $3 \times 3$ identity matrix, then $\Omega=0$. There are two cases for which the corresponding Jacobi form is not saturated; namely, when $B=\operatorname{diag}\left(\lambda_{0}, \lambda_{0}, \lambda_{2}\right)$, and when $B$ has one eigenvalue and one $2 \times 2$ Jordan block. In both these cases the corresponding saturated equation has only linear solutions, albeit infinitely many of them. The two remaining cases correspond to saturated forms whose matrices have Jordan canonical forms of one of the following types:

$$
\left[\begin{array}{ccc}
\lambda_{0} & 0 & 0 \\
1 & \lambda_{0} & 0 \\
0 & 0 & \lambda_{2}
\end{array}\right] \text { and }\left[\begin{array}{ccc}
\lambda_{1} & 0 & 0 \\
1 & \lambda_{1} & 0 \\
0 & 1 & \lambda_{1}
\end{array}\right] \text {, }
$$

where $\lambda_{0} \neq \lambda_{2}$. The form corresponding to the first matrix above has only two linear solutions, one for each eigenvector. The second matrix is more interesting. Writing $\Omega$ in the coordinates for which its canonical matrix is given above, we get

$$
\Omega=\left(2 z x-y^{2}\right) d x-\frac{1}{2} x d\left(2 z x-y^{2}\right),
$$

which has the line $x=0$ and the conics $y^{2}-2 z x=c x^{2}$ for algebraic solutions, where $c \in \mathbb{C}$. Note that, since in this last case $\chi_{\Omega}$ has only one eigenvalue, the change of variables that turns the canonical matrix of $\Omega$ into its Jordan canonical form can be computed over $\mathbb{K}$. Thus, the coordinates $x, y$, and $z$ that appear in (4.4) can be easily found.

\section{REFERENCES}

[1] W. W. Adams and P. Loustaunau, An introduction to Gröbner bases, Grad. Stud. in Math., vol. 3, American Mathematical Society (1994). MR1287608 (95g:13025)

[2] A. M. Cohen, Gröbner bases, an introduction, in Some Tapas of Computer Algebra, A. M. Cohen, H. Cuypers and H. Sterk (eds), Springer (1999). MR.1679917(99k:00038) 
[3] S. C. Coutinho, Indecomposable non-holonomic D-modules in dimension 2, Proc. Edinb. Math. Soc. (2) 46 (2003), 341-355. MR1998565 (2004k:16068)

[4] S. C. Coutinho and L. Menasché Schechter, Algebraic solutions of holomorphic foliations: An algorithmic approach, J. Symbolic Comput. 41 (2006), 603-618. MR2209167 (2007b:32050)

[5] G. Darboux, Mémoire sur les équations différentielles algébriques du $\mathrm{I}^{\mathrm{O}}$ ordre et du premier degré, Bull. des Sc. Math. (Mélanges) (1878), 60-96, 123-144, 151-200.

[6] G.-M. Greuel and G. Pfister, A Singular introduction to commutative algebra, Springer (2002). MR 1930604 (2003k:13001)

[7] G.-M. Greuel, G. Pfister, and H. Schönemann. Singular 2.0.5. A Computer Algebra System for Polynomial Computations. Centre for Computer Algebra, University of Kaiserslautern (2001). http://www. singular.uni-kl.de.

[8] E. L. Ince, Ordinary Differential Equations, Dover (1944). MR0010757 (6:65f)

[9] C. Jacobi, De Integratione Aequationes Differentiallis $\left(A+A^{\prime} x+A^{\prime \prime} y\right)(x d y-y d x)-(B+$ $\left.\left.B^{\prime} x+B^{\prime \prime} y\right) d y+\left(C+C^{\prime} x+C^{\prime \prime} y\right) d x\right)=0, \mathrm{~J}$. für die reine und angewandte Mathematik (1842), pp. 1-4, and Ges. Werke, vol. 4, pp. 256-262.

[10] C. Jordan, Cours d'Analyse de l'École Polytechnique, t. III, 3e éd., Gauthier-Villars, (1915). MR.1188188

[11] J. P. Jouanolou, Equations de Pfaff algébriques, Lect. Notes in Math., 708, Springer-Verlag (1979). MR 537038 (81k:14008)

[12] H. Tsai and U. Walther, Computing homomorphisms between holonomic D-modules. Effective methods in rings of differential operators, J. Symbolic Comput. 32 (2001), 597-617. MR.1866706 (2002k:16051)

Departamento de Ciência da Computação, Instituto de Matemática, Universidade Federal do Rio de Janeiro, P.O. Box 68530, 21945-970 Rio de Janeiro, RJ, Brazil - and - Programa de Engenharia de Sistemas e Computação, COPPe, UfrJ, PO Box 68511, 21941-972, Rio de Janeiro, RJ, Brazil

E-mail address: collier@impa.br

Departamento de Ciência da Computação, Instituto de Matemática, Universidade Federal do Rio de Janeiro, P.O. Box 68530, 21945-970 Rio de Janeiro, RJ, Brazil - and - Programa de Engenharia de Sistemas e Computação, COPPe, UfrJ, PO Box 68511, 21941-972, Rio de JANeiro, RJ, BRAZIL

E-mail address: marcossferreira@gmail.com 